\subsection{OPEN CHROMATIN ANALYSES INFORM FUNCTIONAL NONCODING GWAS VARIANTS IN HIPSC MODEL OF MENTAL DISORDERS}

\author{
Jubao Duan*,1 \\ ${ }^{1}$ NorthShore University Health System/University of Chicago
}

Background: Neuropsychiatric disorders, including schizophrenia (SZ), afflict a significant fraction of the population. Recent genome-wide association studies (GWAS) under the framework of the Psychiatric Genomics Consortium (PGC), along with large-scale sequencing efforts, have identified a plethora of disease risk loci with common and/or rare risk variants. Translating these exciting genomic findings into causation and disease biology offers the promise of developing more tailored therapies in psychiatry. However, understanding the disease biology underlying most GWAS findings remains challenging: (1) The paucity of disease-relevant biological materials for assaying molecular and cellular phenotypes associated with risk loci; (2) Most disease variants lie within poorly-annotated noncoding parts of the genome for which functional interpretation is challenging; and (3) Each locus often contains many genes/variants equivalently associated with the disease due to linkage disequilibrium, and it is difficult to identify which are the likely causal gene/variant. Human neurons derived from induced pluripotent stem cells (iPSCs), both monolayer cultures (2D model) and the emerging brain organoids (3D model), provide a promising alternative to human brains for recapitulating cellular phenotypes relevant to psychiatric disorders. CRISPR/Cas9 editing further strengthens the utility of these models by enabling the generation of isogenic lines with essentially the same genetic background on which allelic effects of a risk variant can be directly compared, thus increasing the sensitivity to detect typically small effects of a GWAS variant.

Methods: To functionally assess the relevance of noncoding sequences in neuropsychiatric disorders, we hypothesized that disease-relevant noncoding sequences likely overlap with cell-specific open chromatin regions (OCRs). We have carried out a genome-wide OCR profiling of excitatory neuronal differentiation from human iPSCs using an Assay for TransposaseAccessible Chromatin by sequencing (ATAC-seq).

Results: We found that OCRs in neurons were enriched SZ risk variants in neural OCRs and can help prioritize putatively functional SZ risk variants that may impact OCRs and consequently, cellular development. At a leading SZ-risk locus flanking MIR137, we further examined the functional effects of a prioritized common GWAS SNP rs1198588 in CRISPR/Cas9-edited hiPSCs, and found that SZ-risk allele of rs1198588 altered MIR137 expression, OCR dynamics and dendrite arborization/ synapse maturation. To systematically identify such disease risk variants that may affect OCR, we further carried out a proof-of-concept analysis of allele-specific open chromatin (ASoC) of in hIPSC-derived neurons. We found that Heterozygous SNPs showing ASoC are more prevalent in neurons than in hiPSCs. Out of the 12 schizophrenia GWAS-implicated SNPs that we found in neuronal OCRs of this single individual, two SNPs showed ASoC and are thus putatively functional: one lies within the 5'-UTR of CHRNA5 (cholinergic receptor, nicotinic, alpha 5) and the other is in the promoter region of VPS45, a Sec1 family gene involved in synaptic transmission. We are currently in the process of replicating the observed landscape of ASoC in iPSC-derived neurons from a larger sample pool.

Discussion: Our study suggests that OCR profiling in a human iPSC model of neuron differentiation can predict functional noncoding sequences that regulate neurodevelopment.

\subsection{MODELING THE CONTRIBUTION OF COMMON VARIANTS TO SCHIZOPHRENIA RISK}

Gabriel Hoffman ${ }^{1}$, Brigham Hartley ${ }^{1}$, Douglas Ruderfer ${ }^{1}$, Judith Rapoport ${ }^{2}$, Pamela Sklar ${ }^{1}$, Kristen Brennand ${ }^{*, 1}$, ${ }^{1}$ Icahn School of Medicine at Mount Sinai; ${ }^{2}$ NIH
Background: Schizophrenia (SZ) is a debilitating psychiatric disorder for which the complex genetic mechanisms underlying the disease state remain unclear. Whereas highly penetrant variants have proven well-suited to human induced pluripotent stem cell (hiPSC)-based models, the power of hiPSC-based studies to resolve the much smaller effects of common variants within the size of cohorts that can be realistically assembled remains uncertain.

Methods: We reprogrammed fibroblasts from SZ patients into hiPSCs and subsequently differentiated these disorder-specific hiPSCs into neural progenitor cells (NPCs) and neurons. Our hiPSC neural cells, from controls and patients with SZ, better resemble fetal rather than adult brain tissue, indicating that hiPSC-based models may be best suited for studies of disease predisposition. At the cellular level, we have previously reported aberrant migration in SZ hiPSC NPCs, together with diminished neuronal connectivity and impaired synaptic function in SZ hiPSC neurons.

Results: We identified microRNA-9 as having significantly downregulated levels and activity in a subset of SZ hiPSC-derived neural progenitor cells NPCs, a finding that was corroborated by a larger replication cohort and further validated by an independent gene-set enrichment analysis of the largest SZ genome-wide association study (GWAS) to date. Overall, this demonstrated a remarkable convergence of independent hiPSC- and genetics-based discovery approaches. In developing this larger case/control SZ hiPSC cohort of hiPSC-derived NPCs and neurons, we identified a variety of sources of variation, but by reducing the stochastic effects of the differentiation process, we observed a significant concordance with two large post mortem datasets.

Discussion: We predict a growing convergence between hiPSC and post mortem studies as both approaches expand to larger cohort sizes. Meanwhile, we have been integrating CRISPR-mediated gene editing, activation and repression technologies with our hiPSC-based neural platform, in order to develop a scalable system for testing the effect of a manipulating the growing number of SZ-associated variants and genes in NPCs, neurons and astrocytes. Altogether, our objective is to understand the cell-type specific contributions of $\mathrm{SZ}$ risk variants to disease predisposition.

\section{IDENTIFYING INDIVIDUALS AT HIGH RISK FOR SCHIZOPHRENIA: LJ SEIDMAN MEMORIAL SYMPOSIUM}

Lynn DeLisi

\section{VA Boston Healthcare System, Brockton Division}

Overall Abstract: Lawrence J. Seidman was born and grew up in New York City. He obtained a PhD from Boston University in Psychology and stayed in Massachusetts to work for many years in the Harvard affiliated Hospitals, such as the VA-Boston Healthcare System, Massachusetts General Hospital and at his untimely passing, he was Professor of Psychology in the Department of Psychiatry at Beth Israel-Deaconess Hospital and the Massachusetts Mental Health Center. He was about to move to a new phase in his career, assuming a position at Children's Hospital, Boston. He was a pioneer in the fields of the neuropsychology of schizophrenia, ADHD and related disorders, of using the tools of cognitive assessments and brain imaging to understand the genetic predisposition for serious mental illness, and in the last several years-prediction of conversion to psychosis in individuals at high risk. He contributed to many multicenter collaborations and had several collaborators world-wide, playing an important role in their work. This symposium is conducted in his honor with contributions from key collaborators on different aspects of his work. Drs. Tyronne Cannon and Elaine Walker will both represent the North American Prodrome Longitudinal Study (NAPLS) consortium by reviewing its findings in brain imaging and cognition. Dr. David Braff will review the work of the Consortium on the Genetics of Schizophrenia (COGS) multicenter collaboration, in which Dr. Seidman led one of its sites, and Dr. TianHong Zhang from Shanghai will present current data from the Shanghai-Boston SHARP collaboration on early detection of 